\title{
THE ROLE OF THE CENTER IN THE THEORY OF DIRECT DECOMPOSITIONS
}

\author{
REINHOLD BAER
}

It has been noticed for a long time that the center plays a fundamental part in the theory of direct decompositions of operator groups and loops. In particular it has been found that the existence of isomorphic refinements of direct decompositions can be assured by imposing conditions which refer solely to the center. ${ }^{1}$ It is the object of the present note to give an explanation for these phenomena by proving quite generally that the validity of the refinement theory in an operator loop is a consequence of the validity of this theory in the center. $^{2}$

In our discussion of operator loops and their direct decompositions we shall use the notations and fundamental definitions which we introduced in a previous paper (Baer [1]) and which are, of course, quite analogous to those customarily used in the theory of operator groups. The refinement theory, however, which we developed recently differs materially from previous statements of the theory, and thus we restate it here in the form best suited to our present purposes.

Definition. If

$$
L=A \oplus B=D \oplus E
$$

are direct decompositions of the $M$-loop $L$ into direct sums of two $M$-subloops, if the refinements

$$
A=A^{\prime} \oplus A^{\prime \prime}, B=B^{\prime} \oplus B^{\prime \prime}, \quad D=D^{\prime} \oplus D^{\prime \prime}, E=E^{\prime} \oplus E^{\prime \prime}
$$

satisfy the conditions

$$
\begin{aligned}
& A^{\prime} \oplus B^{\prime}=B^{\prime} \oplus D^{\prime}=D^{\prime} \oplus E^{\prime}=E^{\prime} \oplus A^{\prime}\left(=L^{\prime}\right), \\
& A^{\prime \prime} \oplus D^{\prime \prime}=D^{\prime \prime} \oplus E^{\prime \prime}=E^{\prime \prime} \oplus B^{\prime \prime}=B^{\prime \prime} \oplus A^{\prime \prime}\left(=L^{\prime \prime}\right),
\end{aligned}
$$

then the decompositions (ii) constitute canonical refinements of the pair (i) of direct decompositions of $L$.

Using this definition our refinement theory may be expressed briefly in the form of the following proposition. 1946.

Presented to the Society, October 25, 1947; received by the editors November 18,

${ }^{1}$ See Baer [1] for bibliographical references and a survey of the pertinent facts, in particular the theorems of Korínek and Kurosh. Numbers in brackets refer to the bibliography at the end of the paper.

${ }^{2}$ A more precise formulation of this statement will be given immediately below. 
Postulate E. If the $M$-subloop $S$ of the $M$-loop $L$ is a direct summand of the $M$-loop $L$, then any pair of direct decompositions of $S$ into two components possesses canonical refinements.

We have to justify our contention that the Postulate $E$ is an adequate expression of a refinement theory.

1. In Baer [1] we have shown that the Postulate $E$ is satisfied under rather general hypotheses; it is valid in particular whenever the hypotheses are valid that have previously been needed for proving refinement theorems.

2. In Baer [1] we have shown that Postulate $E$ implies the existence of exchange isomorphic refinements to any two given direct decompositions of $L$ into a finite number of direct summands. Here we say that the direct decompositions

$$
L=A(1) \oplus \cdots \oplus A(n) \text { and } L=B(1) \oplus \cdots \oplus B(m)
$$

are exchange isomorphic, if $m=n$ and if there exists a permutation $i \rightarrow i^{\prime}$ of the integers from 1 to $n$ such that

$$
\begin{gathered}
L=A(1) \oplus \cdots \oplus A(i-1) \oplus B\left(i^{\prime}\right) \\
\oplus A(i+1) \oplus \cdots \oplus A(n) \quad \text { for every } i .
\end{gathered}
$$

It is easy to verify, and has been shown in Baer [1], that this implies the center isomorphy of $A(i)$ and $B\left(i^{\prime}\right)$ for every $i$.

3. The Postulate $\mathrm{E}$ constitutes nothing but the requirement that decompositions of the special form $L=A \oplus B \oplus F=D \oplus E \oplus F$ with $A \oplus B=D \oplus E$ possess refinements that are exchange isomorphic in a very strict sense.

The principal result that we are going to obtain may now be stated in a more precise form than in the introductory paragraph:

Postulate $\mathrm{E}$ is satisfied by the $M$-loop $L$ whenever it is satisfied by the $M$-center of $L$.

(The center of $L$ consists, as usual, of all the elements in $L$ which commute with every element in $L$ and which associate with every pair of elements in $L$; and the $M$-center $M$ of $L$ is the uniquely determined greatest $M$-subgroup of the center of $L$, that is, the $M$-center is the sum of all the admissible subgroups of the center of $L.)^{3}$

Notation. If $S$ is an $M$-subloop of the $M$-loop $L$, then $Z(S)$ is the $M$-center of $S$.

LEMma. If

${ }^{3}$ It will become apparent in the course of our discussion that we could have substituted for $Z(L)$ a certain, generally proper, $M$-subgroup of $Z(L)$. 


$$
L=A \oplus B=D \oplus E
$$

are direct decompositions of the $M$-loop $L$, and if the decompositions

$$
A=A^{\prime} \oplus A^{\prime \prime}, \quad B=B^{\prime} \oplus B^{\prime \prime}, \quad D=D^{\prime} \oplus D^{\prime \prime}, \quad E=E^{\prime} \oplus E^{\prime \prime}
$$

are canonical refinements of the pair (1) of direct decompositions of $L$, then

$$
\begin{array}{ll}
A^{\prime}=A \cap\left[D \oplus Z\left(E^{\prime}\right)\right], & A^{\prime \prime}=A \cap\left[E \oplus Z\left(D^{\prime \prime}\right)\right], \\
B^{\prime}=B \cap\left[E \oplus Z\left(D^{\prime}\right)\right], & B^{\prime \prime}=B \cap\left[D \oplus Z\left(E^{\prime \prime}\right)\right], \\
D^{\prime}=D \cap\left[A \oplus Z\left(B^{\prime}\right)\right], & D^{\prime \prime}=D \cap\left[B \oplus Z\left(A^{\prime \prime}\right)\right], \\
E^{\prime}=E \cap\left[B \oplus Z\left(A^{\prime}\right)\right], & E^{\prime \prime}=E \cap\left[A \oplus Z\left(B^{\prime \prime}\right)\right] .
\end{array}
$$

Proof. It follows from the definition of canonical refinements that

$$
\begin{aligned}
& A^{\prime} \oplus B^{\prime}=B^{\prime} \oplus D^{\prime}=D^{\prime} \oplus E^{\prime}=E^{\prime} \oplus A^{\prime}\left(=L^{\prime}\right), \\
& A^{\prime \prime} \oplus D^{\prime \prime}=D^{\prime \prime} \oplus E^{\prime \prime}=E^{\prime \prime} \oplus B^{\prime \prime}=B^{\prime \prime} \oplus A^{\prime \prime}\left(=L^{\prime \prime}\right) .
\end{aligned}
$$

From $E^{\prime} \oplus D^{\prime}=E^{\prime} \oplus A^{\prime}$ one deduces that there exists to every element $a$ in $A^{\prime}$ one and only one element $d$ in $D^{\prime}$ such that $a \equiv d$ modulo $E^{\prime}$. There exists one and only one element $e$ in $L$ such that $a=d+e$; and it follows from $a \equiv d$ modulo $E^{\prime}$ that $e$ belongs to $E^{\prime}$. But the mapping of $a$ in $A^{\prime}$ upon $d$ in $D^{\prime}$ is known to be a center isomorphism; and thus $e$ actually belongs to $Z\left(E^{\prime}\right)$. Thus we have shown that $A^{\prime} \leqq D^{\prime}$ $\oplus Z\left(E^{\prime}\right)$; and from $D^{\prime \prime} \oplus E^{\prime \prime}=D^{\prime \prime} \oplus A^{\prime \prime}$ one deduces likewise that $A^{\prime \prime} \leqq E^{\prime \prime} \oplus Z\left(D^{\prime \prime}\right)$. From these inequalities one infers immediately that

$$
\begin{aligned}
& A^{\prime} \leqq A \cap\left[D^{\prime} \oplus Z\left(E^{\prime}\right)\right] \leqq A \cap\left[D \oplus Z\left(E^{\prime}\right)\right] \leqq A \cap\left[D \oplus E^{\prime}\right] \\
& A^{\prime \prime} \leqq A \cap\left[E^{\prime \prime} \oplus Z\left(D^{\prime \prime}\right)\right] \leqq A \cap\left[E \oplus Z\left(D^{\prime \prime}\right)\right] \leqq A \cap\left[E \oplus D^{\prime \prime}\right] .
\end{aligned}
$$

It follows from (4), (2), (1) that

$$
L=L^{\prime} \oplus L^{\prime \prime}=D^{\prime} \oplus E^{\prime} \oplus D^{\prime \prime} \oplus A^{\prime \prime}=D \oplus E^{\prime} \oplus A^{\prime \prime},
$$

implying that $A^{\prime \prime} \cap\left(D \oplus E^{\prime}\right)=0$. Consequently it follows from $A^{\prime} \leqq A \cap\left(D \oplus E^{\prime}\right) \leqq A=A^{\prime} \oplus A^{\prime \prime}$ that

$$
\begin{aligned}
A \cap\left(D \oplus E^{\prime}\right) & =A^{\prime} \oplus\left[A^{\prime \prime} \cap A \cap\left(D \oplus E^{\prime}\right)\right] \\
& =A^{\prime} \oplus\left[A^{\prime \prime} \cap\left(D \oplus E^{\prime}\right)\right]=A^{\prime}
\end{aligned}
$$

and $A \cap\left(E \oplus D^{\prime \prime}\right)=A^{\prime \prime}$ is shown likewise. Hence the inequalities (5) are actually equalities, proving the first two equations (3) of the

${ }^{4}$ This fact as well as many related assertions are very well known in the theory of groups; a verification for loops may be found in various places, for example Baer [1]. 
lemma; the rest is verified likewise. We note that we have proved slightly more than we intended to prove, namely the validity of the following equations:

$$
\begin{aligned}
& A^{\prime}=A \cap\left[D^{\prime} \oplus Z\left(E^{\prime}\right)\right]=A \cap\left[D \oplus Z\left(E^{\prime}\right)\right]=A \cap\left[D \oplus E^{\prime}\right] \\
& A^{\prime \prime}=A \cap\left[E^{\prime \prime} \oplus Z\left(D^{\prime \prime}\right)\right]=A \cap\left[E \oplus Z\left(D^{\prime \prime}\right)\right]=A \cap\left[E \oplus D^{\prime \prime}\right]
\end{aligned}
$$

The following fact is easily verified $:^{4}$ If $L=A(1) \oplus \cdots \oplus A(n)$ is a direct decomposition of the $M$-loop $L$, then $Z(L)=Z(A(1)) \oplus \cdots$ $\oplus Z(A(n))$ is a direct decomposition of the $M$-center of $L$. The latter decomposition will be referred to as the center decomposition induced by the former decomposition.

THEOREM 1. Two canonical refinements of the pair $L=A \oplus B=D \oplus E$ of direct decompositions of the $M$-loop $L$ are equal if, and only if, they induce the same center decompositions.

This is an almost immediate consequence of the equations (3) of the preceding lemma.

TheOREM 2. Suppose that (1) are direct decompositions of the M-loop $L$, that

$$
Z(L)=Z(A) \oplus Z(B)=Z(D) \oplus Z(E)
$$

are the center decompositions induced by (1), and that

$$
\begin{array}{ll}
Z(A)=A^{*} \oplus A^{* *}, & Z(B)=B^{*} \oplus B^{* *}, \\
Z(D)=D^{*} \oplus D^{* *}, & Z(E)=E^{*} \oplus E^{* *}
\end{array}
$$

are refinements of the decompositions (Z.1). Then the refinements (Z.2) are canonical refinements of (Z.1) if, and only if, they are induced in the $M$-center $Z(L)$ of $L$ by canonical refinements of (1).

Proof. It is almost obvious that canonical refinements of (1) induce in the center canonical refinements of (Z.1). Thus the refinements (Z.2) are certainly canonical refinements of (Z.1) if they are induced in the center by canonical refinements of (1).

Assume now that the refinements (Z.2) are canonical refinements of (Z.1). Then we infer from the definition that

$$
\begin{aligned}
Z^{*} & =A^{*} \oplus B^{*}=B^{*} \oplus D^{*}=D^{*} \oplus E^{*}=E^{*} \oplus A^{*} \\
Z^{* *} & =A^{* *} \oplus D^{* *}=D^{* *} \oplus E^{* *}=E^{* *} \oplus B^{* *}=B^{* *} \oplus A^{* *}
\end{aligned}
$$

Clearly $Z(L)=Z^{*} \oplus Z^{* *}$. Following the suggestions inherent to the lemma we define: 


$$
\begin{array}{ll}
A^{\prime}=A \cap\left(D \oplus E^{*}\right), & A^{\prime \prime}=A \cap\left(E \oplus D^{* *}\right), \\
B^{\prime}=B \cap\left(E \oplus D^{*}\right), & B^{\prime \prime}=B \cap\left(D \oplus E^{* *}\right), \\
D^{\prime}=D \cap\left(A \oplus B^{*}\right), & D^{\prime \prime}=D \cap\left(B \oplus A^{* *}\right), \\
E^{\prime}=E \cap\left(B \oplus A^{*}\right), & E^{\prime \prime}=E \cap\left(A \oplus B^{* *}\right) .
\end{array}
$$

It is clear that they are all normal $M$-subloops of $L$. In order to prove that they constitute the desired canonical refinements of (1) we need the $M$-endomorphisms $\alpha, \beta$, and $\delta, \epsilon$ which are uniquely determined by the requirements

$$
\begin{array}{llll}
x \alpha=x \text { for } x \text { in } A, & B \alpha=0, & x \beta=x \text { for } x \text { in } B, & A \beta=0 ; \\
x \delta=x \text { for } x \text { in } D, & E \delta=0, & x \epsilon=x \text { for } x \text { in } E, & D \epsilon=0 .
\end{array}
$$

These endomorphisms induce $e^{4}$ the $M$-center $Z(L)$ of $L$ endomorphisms with completely analogous properties which we designate by the same symbols.

From $B^{*} \oplus A^{*}=B^{*} \oplus D^{*}$ it follows that there exists to every element $x$ in $D^{*}$ one and only one element $y$ in $A^{*}$ such that $x \equiv y$ modulo $B^{*}$; and it is easily seen that $y=x \alpha$. One verifies now that $\alpha$ induces an isomorphism of $D^{*}$ upon $A^{*}$; and in this way one proves the following contentions:4

an isomorphism of $D^{*}$ upon $A^{*}$ and of $E^{* *}$ upon $A^{* *}$ is induced by $\alpha$, an isomorphism of $E^{*}$ upon $B^{*}$ and of $D^{* *}$ upon $B^{* *}$ is induced by $\beta$, an isomorphism of $A^{*}$ upon $D^{*}$ and of $B^{* *}$ upon $D^{* *}$ is induced by $\delta$, an isomorphism of $B^{*}$ upon $E^{*}$ and of $A^{* *}$ upon $E^{* *}$ is induced by $\epsilon$. We prove first:

$$
A^{\prime} \cap A^{\prime \prime}=B^{\prime} \cap B^{\prime \prime}=D^{\prime} \cap D^{\prime \prime}=E^{\prime} \cap E^{\prime \prime}=0 .
$$

If the element $x$ belongs to the cross cut of $A^{\prime}$ and $A^{\prime \prime}$, then $x$ is in $A$ and so $x \beta=0$. Since $x$ is in $A^{\prime}$, there exist uniquely determined elements $r, s$ in $D$ and $E^{*}$ respectively such that $x=r+s$, and since $x$ is in $A^{\prime \prime}$, there exist elements $u, v$ in $D^{* *}$ and $E$ respectively such that $x=u+v$. Since $r$ and $u$ are in $D, s$ and $v$ are in $E$, and since $L=D \oplus E$, it follows that $r=u$ is in $D^{* *}$ and $s=v$ is in $E^{*}$. Thus $x$ belongs to $D^{* *} \oplus E^{*}$. But $\beta$ induces an isomorphism of $D^{* *} \oplus E^{*}$ upon $B$, by (5); and $x \beta=0$. Hence $x=0$, proving $A^{\prime} \cap A^{\prime \prime}=0$; and the remainder of (6) is verified likewise.

$$
A=A^{\prime} \oplus A^{\prime \prime}, \quad B=B^{\prime} \oplus B^{\prime \prime}, \quad D=D^{\prime} \oplus D^{\prime \prime}, \quad E=E^{\prime} \oplus E^{\prime \prime} .
$$

It follows from (6) that the compositum of $A^{\prime}$ and $A^{\prime \prime}$ is their 
direct sum; and hence it follows from (4) that

$$
A^{\prime} \oplus A^{\prime \prime} \leqq A \text {. }
$$

It has been shown elsewhere (Baer [1]) that $\alpha \delta \beta$ maps $L$ into part of its $M$-center. Consider an element $x$ in $A$. Then $x=x \alpha$ and $x \delta \beta=x \alpha \delta \beta$ is therefore an element in $Z(L)$. But $x \delta \beta$ certainly belongs to $L \beta=B$. Hence $x \delta \beta$ is an element in $Z(B)=B^{*} \oplus B^{* *}$; and thus there exist uniquely determined elements $b^{*}$ and $b^{* *}$ in $B^{*}$ and $B^{* *}$ respectively such that $x \delta \beta=b^{*}+b^{* *}$. We infer from (5) the existence of uniquely determined elements $e^{*}$ and $d^{* *}$ in $E^{*}$ and $D^{* *}$ respectively such that $e^{*} \beta=b^{*}$ and $d^{* *} \beta=b^{* *}$. Since $x \delta$ belongs to $D$ and $x \in$ belong to $E$, there exist uniquely determined elements $d$ and $e$ in $D$ and $E$ respectively such that $x \delta=d+d^{* *}$ and $e=x \epsilon+e^{*}$. There exist finally uniquely determined elements $x^{\prime}$ and $x^{\prime \prime}$ such that $x^{\prime}+e^{*}=d$ and $x^{\prime \prime}=e+d^{* *}$. It is clear that $x^{\prime}$ is in $D \oplus E^{*}$ and that $x^{\prime \prime}$ is in $E \oplus D^{* *}$. Furthermore

$$
\begin{aligned}
b^{*}+b^{* *} & =x \delta \beta=\left(d+d^{* *}\right) \beta=d \beta+d^{* *} \beta=\left(x^{\prime}+e^{*}\right) \beta+b^{* *} \\
& =\left(x^{\prime} \beta+b^{*}\right)+b^{* *}
\end{aligned}
$$

so that $x^{\prime} \beta=0$. Hence $x^{\prime}$ is in $A$ and therefore in $A^{\prime}$. Likewise we infer from $x \beta=0$ and the fact that $b^{*}$ and $b^{* *}$ belong to the center that

$$
\begin{aligned}
x^{\prime \prime} \beta & =\left(e+d^{* *}\right) \beta=e \beta+d^{* *} \beta=\left(x \epsilon+e^{*}\right) \beta+d^{* *} \beta \\
& =\left(x \in \beta+b^{*}\right)+b^{* *}=x \in \beta+\left(b^{*}+b^{* *}\right) \\
& =x \in \beta+x \delta \beta=x \beta=0,
\end{aligned}
$$

proving that $x^{\prime \prime}$ belongs to $A$ and therefore to $A^{\prime \prime}$. Considering finally that $e^{*}$ and $d^{* *}$ belong to the center we find that

$$
\begin{aligned}
x+e^{*} & =(x \delta+x \epsilon)+e^{*}=x \delta+\left(x \epsilon+e^{*}\right)=\left(d+d^{* *}\right)+e \\
& =\left(\left(x^{\prime}+e^{*}\right)+d^{* *}\right)+e=\left(x^{\prime}+e^{*}\right)+\left(e+d^{* *}\right) \\
& =\left(x^{\prime}+e^{*}\right)+x^{\prime \prime}=\left(x^{\prime}+x^{\prime \prime}\right)+e^{*}
\end{aligned}
$$

or $x=x^{\prime}+x^{\prime \prime}$ so that $x$ belongs to $A^{\prime} \oplus A^{\prime \prime}$, proving $A=A^{\prime} \oplus A^{\prime \prime}$; the other contentions (7) are verified likewise.

$$
\begin{array}{ll}
A^{*} \leqq A^{\prime} \leqq D^{\prime} \oplus E^{*}, & A^{* *} \leqq A^{\prime \prime} \leqq D^{* *} \oplus E^{\prime \prime}, \\
B^{*} \leqq B^{\prime} \leqq E^{\prime} \oplus D^{*}, & B^{* *} \leqq B^{\prime \prime} \leqq E^{* *} \oplus D^{\prime \prime}, \\
D^{*} \leqq D^{\prime} \leqq A^{\prime} \oplus B^{*}, & D^{* *} \leqq D^{\prime \prime} \leqq A^{* *} \oplus B^{\prime \prime}, \\
E^{*} \leqq E^{\prime} \leqq B^{\prime} \oplus A^{*}, & E^{* *} \leqq E^{\prime \prime} \leqq B^{* *} \oplus A^{\prime \prime} .
\end{array}
$$

Clearly $A^{*} \leqq A \cap\left(D+A^{*}\right) \leqq A \cap\left(D+Z^{*}\right)=A \cap\left(D+D^{*}+E^{*}\right)$ $=A \cap\left(D \oplus E^{*}\right)=A^{\prime} ; A^{* *} \leqq A^{\prime \prime}$, and so on, are verified likewise. Every 
element in $A^{\prime}$ has, by (4), the form $a=d+e^{*}$ for $a$ in $A, d$ in $D$ and $e^{*}$ in $E^{*}$. Thus $0=a \beta=d \beta+e^{*} \beta$. It follows from (5) that $e^{*} \beta$ belongs to $E^{*} \beta=B^{*}$, proving that $d \beta$ is in $B^{*}$. Hence $d=d \alpha+d \beta$ belongs to the cross cut $D^{\prime}$ of $D$ and $A \oplus B^{*}$, proving that $a$ is in $D^{\prime} \oplus E^{*}$ or $A^{\prime} \leqq D^{\prime} \oplus E^{*}$; and the remaining inequalities (8) are verified in a like manner.

$$
\begin{aligned}
& A^{\prime} \oplus B^{\prime}=B^{\prime} \oplus D^{\prime}=D^{\prime} \oplus E^{\prime}=E^{\prime} \oplus A^{\prime}, \\
& A^{\prime \prime} \oplus D^{\prime \prime}=D^{\prime \prime} \oplus E^{\prime \prime}=E^{\prime \prime} \oplus B^{\prime \prime}=B^{\prime \prime} \oplus A^{\prime \prime} .
\end{aligned}
$$

Since $A^{\prime} \leqq A$ and $B^{\prime} \leqq B$, we may infer $A^{\prime} \cap B^{\prime}=0$ from $A \cap B=0$; $D^{\prime} \cap E^{\prime}=0$ is seen likewise. Furthermore we have

$$
B^{\prime} \cap D^{\prime}=B \cap\left(A \oplus B^{*}\right) \cap\left(E \oplus D^{*}\right) \cap D=B^{*} \cap D^{*}=0
$$

by (3); $E^{\prime} \cap A^{\prime}=0$ is verified likewise.

Now we deduce from (3) and (8) that

$$
\begin{aligned}
A^{\prime} \oplus B^{\prime} & \leqq D^{\prime}+E^{*}+B^{\prime} \leqq D^{\prime}+D^{*}+E^{*}+B^{\prime} \\
& \leqq D^{\prime}+D^{*}+B^{*}+B^{\prime} \leqq D^{\prime} \oplus B^{\prime} \\
& \leqq D^{\prime}+E^{\prime}+D^{*} \leqq D^{\prime} \oplus E^{\prime} \\
& \leqq A^{\prime}+B^{*}+E^{\prime} \leqq A^{\prime}+A^{*}+B^{*}+E^{\prime} \\
& \leqq A^{\prime}+A^{*}+E^{*}+E^{\prime} \leqq A^{\prime} \oplus E^{\prime} \\
& \leqq A^{\prime}+B^{\prime}+A^{*} \leqq A^{\prime} \oplus B^{\prime},
\end{aligned}
$$

and the first set of equalities (9) is an immediate consequence of this series of inequalities. The second set of equations (9) is proved likewise.

The normal $M$-subloops, defined by (4), constitute by (7) and (9) canonical refinements of the decompositions (1). These refinements induce in the $M$-center $Z(L)$ of $L$ just the refinements (Z.2) of (Z.1), since, by (8), we have for instance $A^{*} \leqq Z\left(A^{\prime}\right), A^{* *} \leqq Z\left(A^{\prime \prime}\right)$, and $A^{*} \oplus A^{* *}=Z(A)=Z\left(A^{\prime} \oplus A^{\prime \prime}\right)=Z\left(A^{\prime}\right) \oplus Z\left(A^{\prime \prime}\right)$. This completes the proof of Theorem 2 .

Corollary 1. The direct decompositions (1) of the M-loop $L$ possess canonical refinements if, and only if, the induced center decompositions (Z.1) possess canonical refinements. Mapping direct decompositions of $L$ upon the induced center decompositions effects a one-to-one correspondence between the canonical refinements of (1) and the canonical refinements of (Z.1).

These important propositions are immediate consequences of Theorems 1 and 2. 
Theorem 3. Postulate $\mathrm{E}$ is satisfied by the $M$-loop $L$ if, and only if, the following condition is satisfied by $Z(L)$ :

(Z.E) If $A \oplus B=D \oplus E$ is a direct summand of the $M$-loop $L$, then the induced direct decompositions $Z(A) \oplus Z(B)=Z(D) \oplus Z(E)$ possess canonical refinements.

This is an almost immediate consequence of the first assertion of Corollary 1.

Corollary 2. Postulate $\mathrm{E}$ is satisfied by the $M$-loop $L$ whenever it is satisfied by the abelian $M$-group $Z(L)$.

This is an immediate consequence of Theorem 3 , since $Z(A) \oplus Z(B)$ is a direct summand of $Z(L)$, whenever $A \oplus B$ is a direct summand of $L$.

It should be noted that we cannot claim the converse of Corollary 2 to be true. For instance, it may very well happen that $L$ is indecomposable, though Postulate $\mathrm{E}$ is not satisfied by $Z(L)$.

Finally the question may be asked whether it might be possible to prove a like theorem for weaker forms of the refinement theory than the one concentrated in Postulate E. An example, due to Kurosh [1], shows the impossibility of substituting center isomorphy for exchange isomorphy. He has constructed a group $G$, an ordinary group generated by four elements, with the property that $G=A \oplus B$ $=D \oplus E$ where $A, B, D, E$ are indecomposable, $E$ is infinite cyclic and neither $A$ nor $B$ is commutative. Thus $G$ possesses direct decompositions into indecomposable components which are not even isomorphic. But the center of $G$ is a free abelian group of rank 2, and any two direct decompositions of such a free abelian group of rank 2 into indecomposable direct summands are center isomorphic, though not exchange isomorphic (in particular the induced center decompositions are not exchange isomorphic).

\section{BIBLIOGRAPHY}

R. BAER

1. Direct decompositions, Trans. Amer. Math. Soc. vol. 62 (1947) pp. 62-98.

A. KUROSH

1. Isomorphisms of direct decompositions II, Bull. Acad. Sci. URSS. Sér. Math. vol. 10 (1946) pp. 47-72.

UNIVERSITY OF ILLINOIS 